\title{
Open
}

\section{CRS-peptides: unique defense peptides of mouse Paneth cells}

\author{
ML Andersson ${ }^{1}$, JMT Karlsson-Sjöberg ${ }^{1}$ and KL-A Pütsep ${ }^{1}$
}

The intestine is the most densely colonized site in both mice and man. Recent data suggest that the intestinal flora is, in part, controlled by antimicrobial substances secreted by the intestinal epithelium. The defense system of the small intestine includes a protective mucus layer, a high turnover of epithelial cells, and a regulated secretion of effector molecules, notably antimicrobial peptides. Human and mouse small intestines share many similarities in their intestinal defense micro-organization, including the secretion of the well-known $\alpha$-defensins. Mice, however, produce an additional unique antimicrobial peptide family, the CRS (cryptdin-related sequences)-peptides, not found in man. This review comprises a detailed presentation of the peptide-based defense of the gut, with specific emphasis on the CRS-peptide family. The first part presents the current knowledge of the CRS-peptide family's biochemical characteristics and nomenclature, and the second part is devoted to the possible role of this family in the homeostasis of the gut.

\section{INTRODUCTION}

The mucosal intestinal system hosts a large number and broad repertoire of "commensal" bacteria that are of particular use for the host so as to modulate nutrient composition and uptake, to produce essential vitamins and amino acids, and to counteract hostile microbial colonization. The composition of the flora has a significant role in the energy balance and may influence an individual's development toward obesity. ${ }^{1,2}$ Other evidence suggests that changes in the microflora are linked to diseases that affect the cardiovascular system and the liver, and to inflammatory bowels diseases, probably in conjunction with host susceptibility parameters. Accumulating data from patients with inflammatory bowels diseases and in particular Crohn's disease, which most commonly affects the terminal ileum and colon, suggest that such parameters include alterations of the mucosal defense. ${ }^{3}$ Interestingly, several of the associations linked to Crohn's disease involve alterations in specialized intestinal epithelial cells, the Paneth cells, which produce an arsenal of antimicrobial peptides and proteins (reviewed in Porter et al. ${ }^{4}$ ). Paneth cells are relatively longlived secretory cells located at the bottom of small intestinal crypts and these cells are renewed from crypt stem cells. ${ }^{5}$ The majority of Paneth cell antimicrobial peptides/proteins are stored in granules at the apical side of the cells, thus facing the lumen. The secretion of Paneth cell granule constituents is a regulated process that may be induced by bacterial stimulus ${ }^{6}$ or neuronal, cholinergic networks. ${ }^{7,8}$ Upon stimulation, these antimicrobial peptides/proteins are released into the crypt lumen as active molecules at concentrations determined to far exceed what is needed to kill bacteria. ${ }^{6}$ We have previously demonstrated that the released peptides also mix with the overlaying mucins and produce a protective antibacterial mucus layer, thus shielding the epithelial cells from the luminal bacteria. ${ }^{9}$ The presence of Paneth cell-derived $\alpha$-defensins in colonic mucus ${ }^{10}$ suggests considerable peptide stability, which indicates biological relevance beyond the peptide production site.

PART I-CHARACTERISTICS AND NOMENCLATURE OF THE CRS-PEPTIDES FAMILY

\section{Paneth cell antimicrobial peptides}

One of the most well-characterized and highly expressed antimicrobial peptide families is that of the $\alpha$-defensins (also named cryptdins in mice: crypt alpha-defensins). Defensins were among the first antimicrobial peptides to be described, and comprise the $\alpha$-, $\beta$-, and the more exotic $\theta$-defensins. The different defensin families are defined by their three intralinked cysteine bonds and $\alpha$-defensins differ from their cousins $\beta$-defensins in the position of the cysteine bond array. ${ }^{11,12}$ $\beta$-Defensins are well represented in many species, whereas $\alpha$-defensins are restricted to rodents and higher eukaryotes. In man, $\alpha$-defensins are mainly produced by granulated cells

${ }^{1}$ Department of Microbiology, Tumor and Cell Biology, Karolinska Institutet, Stockholm, Sweden. Correspondence: ML Andersson (mats.andersson.2@ki.se) 
such as the Paneth cells and neutrophils, while their synthesis in mice is limited to the Paneth cells of the small intestine. Intestinal $\alpha$-defensins are translated from two-exon genes to a pre-pro protein, where the propart is derived from the first exon and the active antibacterial peptide is coded by the second exon. ${ }^{13}$ Out of the peptides that have been isolated at measurable levels from the mouse small intestine, ${ }^{14-16}$ the $\alpha$-defensins $1-3$, and 6 share with each other a strong amino-acid residue homology, whereas the $\alpha$-defensins 4 and 5 do less so. Many of the mRNA sequences reported represent variant sequences of the $\alpha$-defensins $1-3$ and 6 group. In addition, approximately $20 \alpha$-defensin pseudogenes have been found that are related to rat myeloid $\alpha$-defensins and may represent unproductive ancestral myeloid mouse $\alpha$-defensins. ${ }^{16}$

The CRS-peptides (cryptdin-related sequences) constitute an additional related large family of antimicrobial peptides, unique to mice. ${ }^{17,18}$ This family of peptides was first described by Ouellette and Lualdi ${ }^{17}$ as a new family of Paneth cell-specific mRNAs and the deduced amino-acid sequences from these transcripts revealed pro-sequences highly similar ( $>95 \%$ ) to those of the $\alpha$-defensins (Figure 1a), hence the name cryptdin-related sequence-peptides, CRS-peptides. The CRS-peptides have since then been further characterized and these investigations have revealed an extensive family of highly homologous defense peptides, which the mouse lineage has invested in for gut protection, as the following paragraphs will show. ${ }^{16,18}$

\section{The primary amino-acid sequence and nomenclature of CRS-peptides}

The primary amino-acid sequences of the bactericidal C-terminal part of CRS-peptides are unusual in their high content of cysteines concomitantly with a high proportion of prolines and basic amino-acid residues. Moreover, the cysteines occur in uneven numbers, which is rare for secreted proteins. The CRS-peptides are divided into two groups based on the peptide sequence in the bactericidal C-terminal part, CRS1C and CRS4C (Figure 2). CRS1C-peptides have 11 cysteines in a Cxx repetitive pattern. CRS4C have 9 cysteines (in one occasion 10 cysteines) in a constrained CPx repetitive pattern.

The CRS4C-peptides can be further divided into subgroups defined by the characteristic amino-acid residue sequences in their antimicrobial region, CRS4C-1 (QxxRxxSxxPS), CRS4C-2 (RxxPxxRxSW), and CRS4C-3 (RxxPxxNxxRR) (Figure 2). Although there are several members in each CRS4C-1-3 group, CRS4C-4, CRS4C-5, and CRS4C-6 are the so far deduced peptides with unique sequences. As per the current nomenclature, two different pro-CRS4C-peptides may have identical C-terminal antimicrobial region but differ in their proparts (Figure 2, top). At an mRNA level, each CRC4C transcript has been designated a prefix ( $a, b, c$, etc.), based on the unique protein sequence of the entire pro-protein even though the antimicrobial CRS-peptide region may be identical. All CRS4C peptides are basic peptides with a net charge of +5 to +7 and an isoelectric point in between 8.5 and 9.3. CRS4C-4 and CRS4C-6 are the least charged peptides (Figure 2).

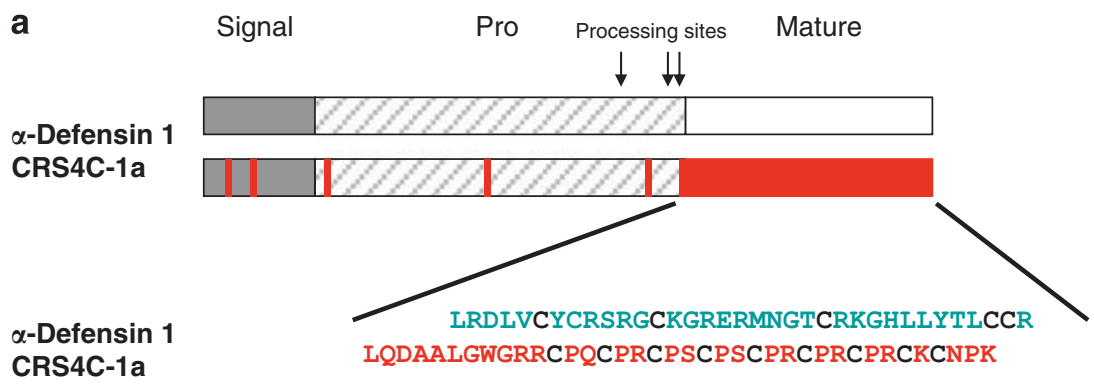

b

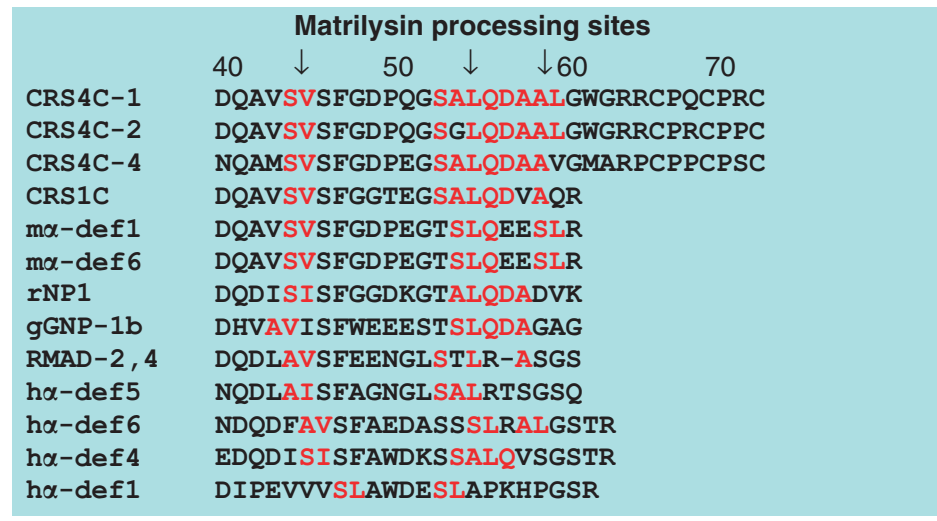

Figure 1 Proteolytic activation of $\alpha$-defensins and cryptdin-related sequences (CRS)-peptides. (a) Schematic presentation of translated mRNA from $\alpha$-defensin 1 and CRS4C-1. Gray denotes signal peptide region, shaded the propart (Pro) region, and white the antimicrobially active peptide region. Red indicates the active peptide region, where there is no homology between $\alpha$-defensin- 1 and CRS4C-1, and below are the amino-acid sequences of the active peptides shown. Arrows indicate the proteolytic processing sites for the MMP7 enzyme. (b) Comparison of CRS-peptide and $\alpha$-defensin amino-acid sequences of the target region for MMP-7 in mouse (m $\alpha)$, rat (rNP1), guinea-pig (gGNP), monkey (RMAD), and man (h $\alpha)$. 


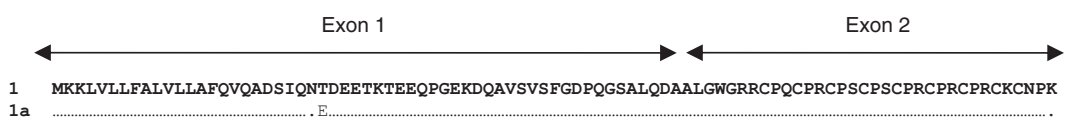

\begin{tabular}{|c|c|c|c|}
\hline & & & IP \\
\hline $4 C-1 a$ & & 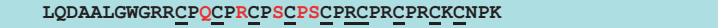 & 9.24 \\
\hline $4 C-1 d$ & & 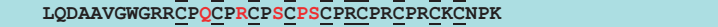 & 9.24 \\
\hline $4 \mathrm{C}-2$ & & 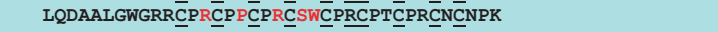 & 9.08 \\
\hline $4 \mathrm{C}-2 \mathrm{~b}$ & & 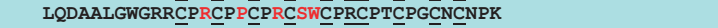 & 8.90 \\
\hline $4 C-3 a$ & & 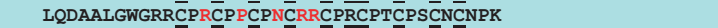 & 9.08 \\
\hline $4 C-3 c$ & & 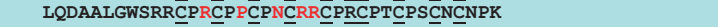 & 9.08 \\
\hline $4 C-3 d$ & & 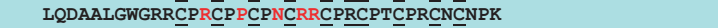 & 9.27 \\
\hline $4 \mathrm{C}-4$ & & 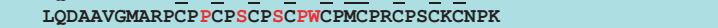 & 8.47 \\
\hline $4 C-5$ & & LQDAAIRRARRCP PCP SCLSCPWCPRCPLCPICKCNPK & 9.06 \\
\hline $4 C-6$ & & LQVSGLGKPPQCPKCPVCSKCPQCPQCPQCPGCPRCNCMTK & 8.79 \\
\hline Conserved & $(1-3)$ & LQDAA GW RRCP $\underline{\mathrm{CP}} \underline{\mathrm{CP}} \underline{\mathrm{C}} \underline{\mathrm{CPR}} \underline{\mathrm{CP}} \underline{\mathrm{CP}} \underline{\mathrm{C}} \underline{\mathrm{CNPK}}$ & \\
\hline $1 \mathrm{C}-1$ & \multicolumn{2}{|r|}{ LQDVAQRRFPWCRKCRVCQKCEVCQKCPVCPTCPQCPKQPLCKERQNKTAITTQAPNTHHKGC } & 9.24 \\
\hline $1 \mathrm{C}-2$ & \multicolumn{2}{|r|}{ 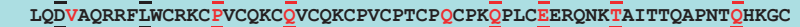 } & 9.00 \\
\hline $1 \mathrm{C}-3$ & \multicolumn{2}{|r|}{ 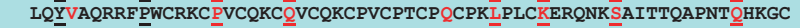 } & 9.34 \\
\hline $1 C-4$ & \multicolumn{2}{|r|}{ 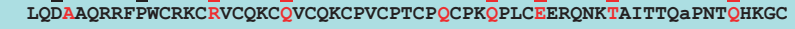 } & 9.13 \\
\hline 1C 5 & \multicolumn{2}{|r|}{ LQDVAQRRFPWCRKCRVCQKC $Q V C Q K C P V C P T C P L C P K Q P L C E E R Q N K T A I T T Q A P N T Q H K G C$} & 9.13 \\
\hline $1 \mathrm{C}-6$ & \multicolumn{2}{|r|}{ LQDVAQRRFPWCRKC PVCQKCQVCQKCPVCPTCPQCPKLPLCKERQNKSAITTQAPNTQHKGC } & 9.24 \\
\hline Conserved & \multicolumn{2}{|r|}{ LQ AQRRF WCRKC VCQKC VCQKCPVCPTCP CPK PLC ERQNK AITTQAPNT HKGC } & \\
\hline
\end{tabular}

Figure 2 Amino-acid sequences of CRS4C and CRS1C peptides. Top: Comparison of two CRS4C-1 pre-pro peptides. Middle: CRS4C peptides 1-4 and predictive processed peptides of CRS4C-4--6. Bottom: CRS1C predictive processed peptides. Predictive processing is displayed for peptides in which mRNA sequences have not been confirmed by detection at a protein level. Numbers presented to the right of the peptide sequence are their respective isoelectric points (IP). Sequences are from Shanahan et al.., ${ }^{16}$ Hornef et al., ${ }^{18}$ Amid et al., ${ }^{19}$ and Huttner and Ouelette. ${ }^{21}$

Strain-specific repertoires of CRS genes exist. The most frequently used mouse strain for construction of knockout mutants and transgenes, the C57BL/6 strain, diverges the most from all the other mouse strains so far analyzed. ${ }^{16,19,20}$ However, the nomenclature does not take into account the mouse strain of origin (Figure 3), which has most likely confused researchers, and therefore a consistent model of nomenclature is needed.

The second main group of CRS-peptides, the CRS1C-peptides, has fewer members, which have been numbered consecutively following their identification: CRS1C-1-6 (Figure 2). The deduced CRS1C-peptides have higher net charge than CRS4C ranging from +7 to +10 , but similar isoelectric values (9.0-9.3; Figure 2). The CRS1C-peptides were initially described in outbred Swiss mice ${ }^{17}$ and later identified in 129/SVJ, as well as $\mathrm{FvB},{ }^{21}$ and more recently in the $\mathrm{C} 57 \mathrm{BL} / 6$ strain ${ }^{19,20}$ (Figure 3). The amino-acid residue variation occurs at 10 of 63 positions, suggesting a higher degree of conservation than what has been found for the CRS4C family.

\section{The defensin-CRS genome cluster}

The mouse $\alpha$-defensin genes (provisionally termed Defcr\#) and CRS genes (provisionally termed Defcr-rs\#) are located on chromosome 8. ${ }^{16,19,22}$ The mouse locus is organized in a $\beta$-defensin$\alpha$-defensin-CRS- $\alpha$-defensin- $\beta$-defensin cluster (Figure 4). ${ }^{16,19}$ Human chromosome 8 displays a similar locus structure; $\beta$-defensins- $\alpha$-defensins- $\beta$-defensins, even though $\beta$-defensins are as well present on other chromosomes. ${ }^{23}$ Genome-wide analysis of the human genome has identified this part of the human chromosome 8 as a hot spot for copy number variations (http://www.sanger.ac.uk/humgen/cnv/), which may affect both disease as well as normal phenotypic variation and may shape tissue transcriptomes. ${ }^{24,25}$

The complete mouse defensin locus is yet to be defined and further analysis of this locus will most likely uncover additional differences among individual mouse lineages. At least 20 different $\alpha$-defensin mRNAs have been identified from different mouse strains. ${ }^{13,16}$ At present, the genomic locus based on the C57BL/6 strain ${ }^{16}$ is better defined compared with the mixed strain genome assembly generated from the Celera/NCBI database. ${ }^{19}$ The C57BL/6 strain genome assembly presents an $\alpha$-defensin locus in which $\alpha$-defensin genes, CRS, and $\alpha$-defensin pseudogenes are intermingled. The C57BL/ 6 strain expresses CRS4C- 6 and several CRS1C peptides. ${ }^{16,20}$ Introns of the CRS4C genes are approximately 95\% identical to each other and share approximately $80 \%$ nucleotide identity with CRS1C genes. However, the introns of CRS-peptides are completely unrelated to the mouse $\alpha$-defensin gene introns, except for the exon-intron junctions. ${ }^{21}$ In fact, the introns of CRS4C and CRS1C are more similar to the introns in rat $\alpha$-defensin genes than to mouse $\alpha$-defensin genes. ${ }^{16}$ Interestingly, all $\alpha$-defensin genes, including pseudogenes, are oriented in the centromer direction, whereas the CRS genes are in the telomere direction. Thus, although the pro-regions of the mouse alpha-defensins and CRS-peptides are highly homologous, the evolutionary relationship between mouse CRS-peptides and $\alpha$-defensins is still enigmatic.

Several of the CRS4C genes are poorly annotated as these genes are predominately expressed in $\mathrm{C} 3 \mathrm{H} / \mathrm{HeJ}, \mathrm{FVB}$, or 129/SVJ strains, whereas CRS1C genes are more frequent in C57BL/6 strain (Figure 3). A summary of the MGI nomenclature to synonyms and corresponding CRS-peptides is presented in Table 1. It is likely that both $\alpha$-defensins and CRS-peptides originate from a limited number of genes that, owing to gene duplication and mutations, result in a set of similar but not identical peptides. Thus, what appears as a plethora of peptides is in fact a result of gene amplification. Such evolution is most likely under positively selection, as has been suggested for $\beta$-defensins. ${ }^{26-28}$ 


\begin{tabular}{|c|c|c|c|c|c|c|c|c|c|c|c|c|c|}
\hline Mouse strain & $1 \mathrm{C}$ & $\begin{array}{c}1 \mathrm{C}- \\
1 / 2 / 3\end{array}$ & $\begin{array}{c}1 C- \\
4 / 5 / 6\end{array}$ & $\begin{array}{l}4 C- \\
1 a\end{array}$ & $\begin{array}{l}4 C- \\
1 b\end{array}$ & $\begin{array}{l}4 C- \\
2\end{array}$ & $\begin{array}{l}4 C- \\
b\end{array}$ & $\begin{array}{l}4 C- \\
3 a\end{array}$ & $\begin{array}{l}4 C- \\
3 c\end{array}$ & $\begin{array}{l}4 C- \\
3 d\end{array}$ & $\begin{array}{l}4 C- \\
3 f\end{array}$ & $\begin{array}{l}4 C- \\
4 / 5\end{array}$ & $\begin{array}{l}4 C- \\
6 \\
\end{array}$ \\
\hline FVB & $x$ & & $x$ & $x$ & $x$ & $x$ & & $x$ & & $x$ & $x$ & & \\
\hline $\mathrm{C} 3 \mathrm{H} / \mathrm{HeN}$ & & & & $x$ & $x$ & $x$ & $x$ & $x$ & $x$ & $x$ & & & \\
\hline $\begin{array}{l}\text { Outbred } \\
\text { Swiss albino }\end{array}$ & $\mathbf{x}$ & & & $\mathbf{X}$ & & & & & & & & & \\
\hline $129 / \mathrm{SvJ}$ & & & & $x$ & $x$ & $x$ & & $x$ & & & & $x$ & \\
\hline C57/BL6 & & $x$ & & & & & & & & & & & $x$ \\
\hline
\end{tabular}

Figure 3 Mouse strain-specific mRNA expression of different CRS4C and CRS1C peptides. Adapted from Karlsson et al. ${ }^{20}$

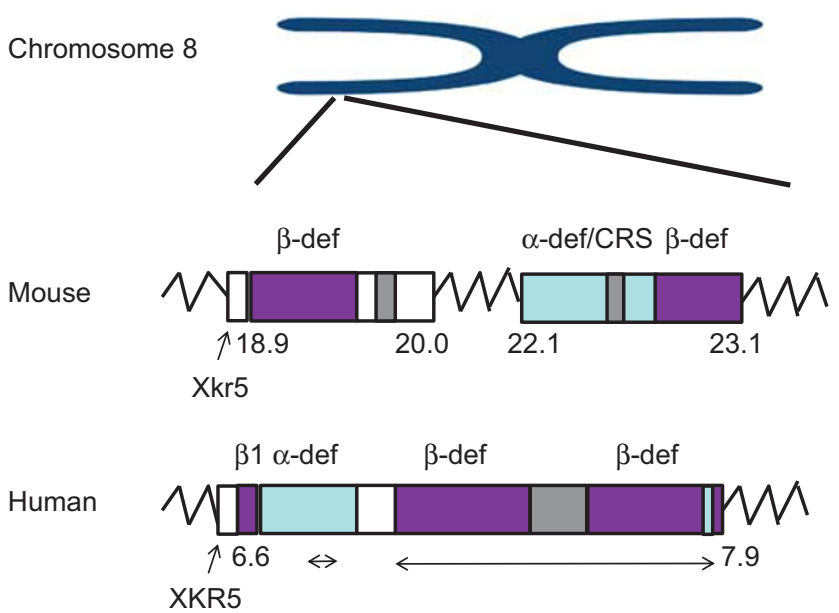

Figure 4 Schematic representation of the defensin gene loci on chromosome 8 in mice and man. Top: Overview of chromosome 8 with the defensin locus marked. Middle: Defensin locus in mice. Bottom: Defensin locus in man. Blue, alpha-defensin region; violet, beta-defensin region; white, non-defensin region; grey, undefined region. Double arrow indicates the area with gene copy number variations. Numbers indicate chromosome location. CRS, cryptdin-related sequences; $\alpha$-def, alphadefensin genes; $\beta$-def, beta defensin genes; X kr5/XKR5, Kell blood group complex subunit-related family member.

\section{Proteolytic activation of Paneth cell peptides}

All mouse $\alpha$-defensins, except $\alpha$-defensin 4 , are proteolytically activated by the enzyme matrix metalloproteinase 7 (MMP7, matrilysin) to release the active bactericidal peptide ${ }^{8}$ (Figure 1a). The $\alpha$-defensin 4 is proteolytically released by MMP7, but then further processed by a trypsin-like enzyme. ${ }^{29}$ The processing site for MMP7 is conserved in rat and guineapig, but has diverged in monkey and man (Figure 1b), most likely coinciding with a shift in processing-enzyme from MMP7 to a Paneth cell-derived trypsin in man. ${ }^{30}$

Among the CRS-peptides, the group of CRS4C-peptides present three potential MMP7 processing sites, $\mathrm{ser}^{43}-\mathrm{val}^{44}$, ala $^{53}-\mathrm{leu}^{54}$, and ala ${ }^{58}-\mathrm{leu}^{59}$ (Figure 1b), ${ }^{18,31,32}$ and indeed three different lengths of CRS4C pro-peptides were identified, suggesting that alternative or sequential processing may occur. ${ }^{8,31}$

\section{Intestinal expression of CRS-peptides and $\alpha$-defensins}

Different mouse lineages produce somewhat different repertoires of $\alpha$-defensins, similar to that of the CRS-peptides. One
Table 1 The mouse genome informatics (MGI) designation of commonly found genes and their peptide products

\begin{tabular}{lll}
\hline MGI & Name & Peptide \\
\hline Defa-rs1 & Defensin alpha-related sequence 1 & CRS1C-2 \\
AY761184 & cDNA sequence AY761184 & CRS1C-3 \\
Defa-rs2 & Defensin alpha-related sequence 2 & CRS4C-1 \\
Defa-rs4 & Defensin alpha-related sequence 4 & CRS4C-1a \\
Defa-rs5 & Defensin alpha-related sequence 5 & CRS4C-1b \\
Defa-rs6 & Defensin alpha-related sequence 6 & CRS4C-1c \\
Defa-rs7 & Defensin alpha-related sequence 7 & CRS4C-2 \\
Defa-rs8 & Defensin alpha-related sequence 8 & CRS4C-2a \\
Defa-rs9 & Defensin alpha-related sequence 9 & CRS4C-3 \\
Defa-rs10 & Defensin alpha-related sequence 10 & CRS4C-4 \\
AY761185 & CDNA sequence AY761185 & CRC4C-6 \\
\hline
\end{tabular}

example is the mouse C57BL/6 strain that does not produce the $\alpha$-defensins 1, 2, 4, 6, and 7, but which are found in 129/SvJ, $\mathrm{C} 3 \mathrm{H} / \mathrm{HeJ}, \mathrm{FvB}, \mathrm{BALB} / \mathrm{c}$, and outbred Swiss mice. Instead, the C57/BL6 strain presents the $\alpha$-defensins 20,21,23,24, and 27, out of which $\alpha$-defensin 20 was previously named Crp4-B6a and $\alpha$-defensin $21 / 22$ was named Crp4-B6b. ${ }^{32}$ Both of these $\alpha$-defensin 4 variants lack three codons between the fourth and fifth cysteine residues, thus changing the classic MMP7 cleavage site in $\alpha$-defensin 20 and $\alpha$-defensin $21 / 22$ and leading to $\mathrm{N}$-terminal elongated peptides. Moreover, there is no $\alpha$-defensin 6 in C57BL/ 6 mice, but instead an $\alpha$-defensin 24 with an $\arg ^{24}$ to $\operatorname{ser}^{24}$ replacement (M Andersson, unpublished).

CRS4C peptide expression in the small intestine is substantial and the concentration is in the same range as that of the $\alpha$-defensins. ${ }^{20}$ CRS4C transcripts have been demonstrated in other tissues as well, such as skin, testis ${ }^{33-35}$ cecum, and thymus, ${ }^{20}$ although the levels of active peptide remain to be established.

CRS1C peptides and $\alpha$-defensins (with the exception of $\alpha$-defensin 4 ) are equally expressed along the small intestine. ${ }^{20}$ However, the production of CRS4C peptides and $\alpha$-defensin 4 is mainly restricted to the distal (ileum) region of the small intestine $^{20}$ (Figure 5). This implies that the CRS4C peptides and $\alpha$-defensin 4 affect the local microbiota in the distal ileum. 

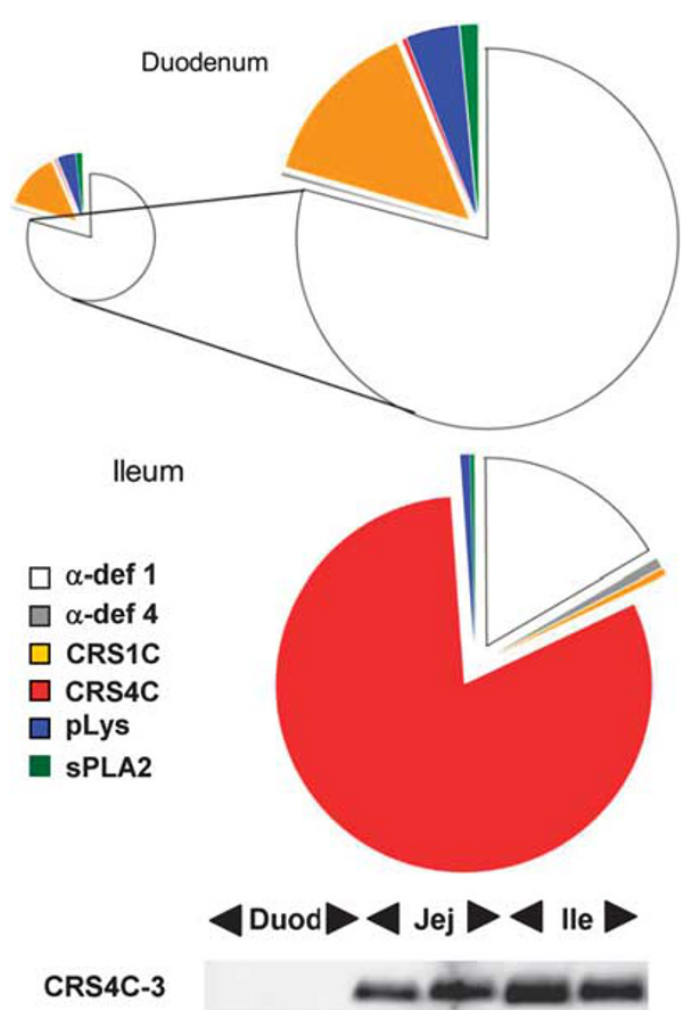

Cryptdin 2

Figure 5 Spatial expression of Paneth cell protein/peptides along the mouse small intestine. Top: Pie chart representation of transcript levels in the duodenum and ileum, respectively. The marked areas are proportional to the total number of mRNA copies of analyzed antimicrobials. Bottom: Protein expression analyzed by immunoblot of extracted tissues. $\alpha$-def, $\alpha$-defensin; Doud, duodenum; lle, ileum; Jej, jejunum; pLys, Paneth cell lysozyme; sPLA2, secreted phospholipase A2. With permission from Karlsson et al. ${ }^{20}$

A similar distribution pattern has been demonstrated in humans, although not as pronounced, where the $\alpha$-defensins HD5 and HD6 coordinately have increased expression from the proximal to distal intestine. ${ }^{36}$ Moreover, increased secretion of HD5 has been noticed, as well as an increase in HD5-expressing intermediate cells and enterocytes, in the crypts of patients who undergo Roux-en- $Y$ gastric bypass surgery, ${ }^{37}$ suggesting an adaptation of host defense to the increased number of bacteria.

Information about factors that regulate $\alpha$-defensin gene activity in the small intestine is still limited. The differentiation of intestinal stem cells and commitment to the Paneth cell lineage is dependent on several transcription factors (reviewed in Hauck et al. ${ }^{38}$ ). The transcription factor Tcf- 4 has a major role in both early differentiation and in the maturation of Paneth cells ${ }^{39}$ following Wnt $/ \beta$-catenin-mediated signalling. ${ }^{40} \mathrm{Tcf}-4$ directs also the expression of defensins and CRS-peptides, as shown for CRS1C, $\alpha$-defensin 1, 4, and 5 in mice, and HD5 and HD6 in humans. ${ }^{39,41,42}$ The $\alpha$-defensin promoter region has multiple Tcf- 4 binding sites that display individual patterns of location in the promoter region for different $\alpha$-defensins. Using promoter constructs of mouse $\alpha$-defensin 5 , it was

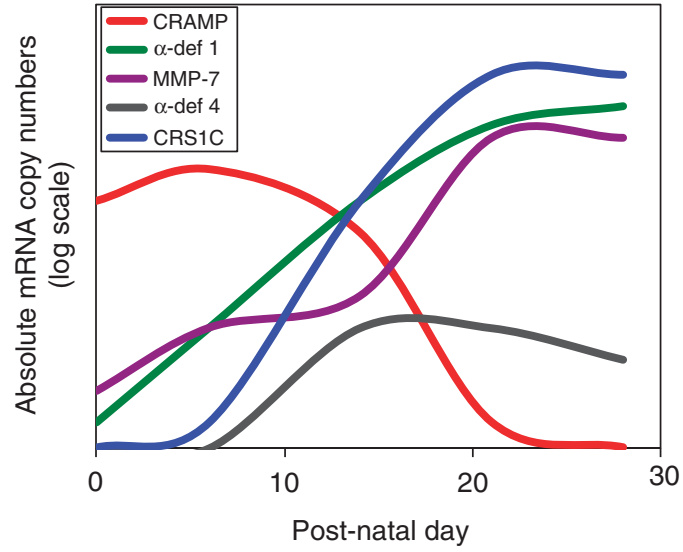

Figure 6 Postnatal developmental regulation of antimicrobial peptide synthesis in the developing mouse ileum. $\alpha$-def, $\alpha$-defensin; CRAMP, mouse cathelicidin; MMP7, matrix metalloproteinase 7. Data obtained from Karlsson et al. ${ }^{20}$ and Menard et al. ${ }^{45}$

demonstrated that the 2,500-base pair upstream region of the start site resulted in transcriptional activity when challenged with Tcf- 4 and activated $\beta$-catenin. ${ }^{39}$ CRS4C genes also present potential Tcf-4-binding sites dispersed in the promoter region (J Karlsson-Sjöberg, unpublished results), but with great variation among the various CRS4C genes. We find it probable that additional/other transcription factors, with a gradually increasing expression pattern throughout the small intestine regulate peptides such as CRS4C and $\alpha$-defensin 4 . Moreover, Paneth cell secretions from heterozygous Tcf- $4(+/-)$ mice (homozygote mice die) showed reduced antibacterial activity against Escherichia coli and Staphylococcus aureus as compared with wild-type mice. ${ }^{42}$ This is in line with an anticipated effect of reduced levels of $\alpha$-defensin 1 and $\alpha$-defensin 4 .

\section{PART II-THE ROLE OFTHE CRS-PEPTIDES AND DEFENSINS INTHE GUT HOMEOSTASIS Developmental regulation of CRS-peptides}

The Paneth cell linage does not appear in the newborn mouse small intestine until postnatal day 7 (P7), with the characteristic morphology of Paneth cells, including granules, and is not fully matured until P28 after birth. ${ }^{43}$ This cell lineage expands extensively during the time period $\mathrm{P} 14-\mathrm{P} 21^{44}$ and this Paneth cell maturation process concords with the weaning period of the mice. Expression of $\alpha$-defensins and CRS-peptides is low or absent during the beginning of the postnatal period (Figure 6). ${ }^{44,45}$ The antimicrobial cathelicidin peptide CRAMP, on the other hand, is constitutively expressed in epithelial intestinal cells during the neonatal period, but absent in isolated small intestinal epithelial cells after day P21. ${ }^{45}$ Between days P12 and P25, a major shift in the repertoire of antibacterial peptides takes place in the small intestine in which production of CRAMP is superseded by $\alpha$-defensins and CRS-peptides. ${ }^{45}$ We found that germ-free mice, similar to conventionally bred mice, lack intestinal epithelial CRAMP expression in adulthood, which precludes an active role of the enteric microbiota in the downregulation of intestinal CRAMP expression. ${ }^{45}$ 
The close temporal association between the loss of CRAMP expression and developmental changes such as the increase in epithelial proliferation, the emergence of crypts, and the appearance of mature Paneth cells indicates a developmentally governed process.

Bacteria are not required for the synthesis of either CRSpeptides or $\alpha$-defensins as germ-free mice produce mature $\alpha$-defensins, as well as CRS-peptides. ${ }^{20,31}$ Thus, most of the peptide repertoire is formed independent of colonization. However, we detected a two- to threefold higher level of CRS-peptides in conventionally reared mice as compared with germ-free mice, ${ }^{20}$ possibly owing to increased Paneth cell numbers following bacterial colonization. ${ }^{46}$ We observed no altered synthesis of CRS-peptides or $\alpha$-defensins in adult mice after oral challenge with the pathogenic bacteria Salmonella typhimurium or Listeria monocytogenes. ${ }^{20}$ Interestingly, mono-association of germ-free mice with the bacterium Bacteroides thetaiotaomicron modifies the genetic program of the Paneth cell lineage and enhances the synthesis of the bactericidal proteins ribonuclease Angiogenin 4 and secreted C-type lectin, RegIII $\gamma$ around 10 -fold. ${ }^{20,47}$ Thus, certain microbes may differentially modulate the production of Paneth cell bactericidal peptides/proteins, which in turn could influence the composition of the evolving intestinal microbiota.

\section{Antimicrobial activity of CRS-peptides and defensins}

An extraordinary feature of CRS-peptides is that they form covalent homo- and heterodimers. ${ }^{18}$ Each peptide contains an odd number of cysteines, and theoretically at least one cysteine in each peptide would be involved in the dimerization. Peptides extracted in the presence of iodo-acetamine to prevent oxidation and cysteine linkage did not present as monomeric CRS4C peptides, suggesting that covalent dimers are formed early during cellular processing. Interestingly, a variant form of a mouse $\beta$-defensin with only five cysteines that also form covalent dimers has been described. ${ }^{48}$ One role of covalent dimerization may be higher or more longlasting antimicrobial activity. Another possibility may be that random dimerization of different CRS4C monomers expands the repertoire of antimicrobial activity. Both of these possibilities seem to be true for CRS4C peptides. ${ }^{18}$ Using isolated peptides from the small intestine, we demonstrated that homo- and heterodimers of CRS-peptides differ in killing activities. For instance, the heterodimer of CRS4C-1a/3a-truncated was three times as active against the Gram-negative S. typhimurium as the CRS4C-3a-truncated/3a-truncated homodimer. The Gram-positive bacterium Streptococcus pyogenes, on the other hand, was two times as sensitive to the CRS4C-1a/1a homodimer as that to the CRS4C-2/3a heterodimer. This capacity of CRSpeptides to form covalent dimers increases, thus, their antimicrobial repertoire, as does the existence of truncated forms of the peptides. ${ }^{18}$ The difference in bacterial killing between different forms of the peptides was confirmed by using synthetic CRSpeptides, and the dimerized peptides were two- to fourfold more potent than their monomeric forms. ${ }^{18}$

The intestinal $\alpha$-defensins target both Gram-positive and Gram-negative bacteria and the antimicrobial spectra of indi- vidual $\alpha$-defensins differ. ${ }^{15,16}$ Similar to what has been found for the CRS-peptides, truncation or elongation of the $\alpha$-defensins alters their activity range. ${ }^{16,49}$ Minor exchanges of residues may have a profound impact on bactericidal activity. For example, $\alpha$-defensins 2 and 3 differ in only one amino-acid position $\left(\mathrm{T}^{10}\right.$ and $\mathrm{K}^{10}$, respectively), but contrary to $\alpha$-defensin $3, \alpha$-defensin 2 does not kill E. coli. The abundantly expressed C57BL/6 mouse-derived $\alpha$-defensin 20 (formerly Crp4-B6a) exhibits less antibacterial activity against $E$. coli ML35 and attenuated S. typhimurium in vitro as compared to the "white mouse" $\alpha$-defensin 4 , although both peptides have similar activity towards L. monocytogenes 104035 and S. aureus 710a. ${ }^{32}$

Thus, CRS4C peptides and $\alpha$-defensins differ in antibacterial spectra and kinetics whereas their bactericidal activities require in both cases concentration in the micromolar range. Taken together, it seems as though the mouse Paneth cells, compared with human Paneth cells, are equipped with a broader repertoire of antimicrobial peptides, ready to control the growth of harmful opportunistic bacteria, as well as commensals of the microflora.

\section{Redox modulation of antibacterial activity}

Contrary to the $\alpha$-defensins, which present considerable antibacterial activity even when reduced, the CRS4C-1 peptide lost most of its activity towards S. typhimurium and L. monocytogenes when reduced and alkylated to open all disulfide bonds. ${ }^{49}$ However, alkylated CRS4C-1 showed increased activity against $E$. coli ML35, thus demonstrating that the selectivity of the peptide is affected by its redox status. Similarly, a recent paper suggests that controlling the redox status of the human antimicrobial peptide $\beta$-defensin 1 is a potent mechanism to regulate its activity. ${ }^{50}$ Previously, human antimicrobial peptide $\beta$-defensin 1 was considered to be a peptide with relative weak antibacterial activity. However, when reduced it acquired increased activity. Reduced cysteines were suggested to be generated physiologically by the thioredoxin system that is co-localized with human antimicrobial peptide $\beta$-defensin $1 .{ }^{50}$ We have previously shown that the human redox system thioredoxin-thioredoxin reductase reduces disulfides in antibacterial peptides such as $\alpha$-defensin HNP3 and NK-lysin. ${ }^{51-53}$ Contrary to the human thioredoxin, the E. coli thioredoxin was not able to reduce the disulfide bonds. Moreover, the host thioredoxin system may not only modulate antimicrobial activity towards a repertoire of bacteria, but also reduce their cytotoxic effect against host cells. ${ }^{52}$

A recently proposed mechanism for Salmonella infection defines intestinal inflammation and the capacity of the bacteria to persist oxidative conditions during translocation through the epithelial barrier as a prerequisite for bacterial success. ${ }^{54}$ Intestinal inflammation leads to the production of reactive oxygen species that react with endogenous luminal sulfur compounds (thiosulfate) to form a new compound, tetrathionate. Tetrathionate in turn can then be utilized by Salmonella as an electron acceptor, which confers growth advantage. ${ }^{54}$ This elegant study also raises the question whether the presence of abundant defensins and CRS-peptides, apart from their antibacterial 
activities, may in addition act as sulfur scavangers owing to their high content of cysteine residues. The activity of antimicrobial peptides is dependent on primary structure, processing, and modifications, which in turn may be affected by environmental conditions such as redox state and salt concentration. Thus, the susceptibility of different mouse strains to various pathogens may, in part, be governed by the repertoire and post-translational modifications of their $\alpha$-defensins and CRS-peptides.

\section{Peptide-based protection of the gut: mouse models for intestinal disease}

Pathological conditions, owing to reduced antimicrobial activity in the proximity of the intestinal epithelial cell layer (Figures $7 \mathbf{a}$ and $\mathbf{b}$ ), may precipitate from different cell abnormalities: (i) inadequate sensing of microbes, (ii) reduced production of antimicrobial components or incorrect processing yielding inactive peptides, and (iii) inadequate granule packing and/or release of granule content.

(i) Paneth cells can directly sense microbial components and release antibacterial substances into the lumen. ${ }^{6}$ Interference with pathways of microbial sensing by ablation of Nod2 (intracellular microbial response receptor protein), MyD88 (intracellular adaptor protein), or Rip2 (intracellular downstream kinase) all generate mice with a reduced capacity of crypt secretions to kill microbes ${ }^{55,56}$ (Figure $7 \mathbf{b}$ ). It has been reported that Nod2 ablation reduces the synthesis of CRS4C-4 to levels comparable to the levels expressed in germ-free mice (fourfold less). ${ }^{55}$ Nod2 exhibits a limited cell expression, but is present in Paneth cells and, importantly, mutations in the NOD2 gene were the first identified genetic risk factor for Crohn's disease. ${ }^{57}$ In mice, Nod2 is essential for temporal development of the commensal flora. ${ }^{58}$ Both Nod2- and Rip2-negative mice are more sensitive to oral infections that may precipitate intestinal inflammation, as compared with wild-type mice. Restoring the crypt antimicrobial function, by introducing the human HD5 gene through crossbreeding with $\mathrm{HD} 5+/+$ mice, reversed the inflammation in Nod2-negative mice. ${ }^{56}$ Thus, HD5 synthesis in Paneth cells overcomes the lack of Nod2-induced mobilization of crypt antimicrobial activity. Future studies are likely to reveal at what level Nod2 does influence Paneth cell endogenous antimicrobial component secretion.

MyD88 is a key player in conveying signals upon recognition of microbial components through a family of membrane-bound microbial pattern recognition receptors, the Toll-like receptors. Paneth cells express low levels of Toll-like receptors 1-3, 5-9 mRNA, ${ }^{59}$ while Toll-like receptor 4 , which is the major lipopolysaccharide signalling target, is absent in Paneth cells. Other enterocytes of the intestinal epithelium express Toll-like receptor 4, but the localization is restricted to their intracellular space. ${ }^{60} \mathrm{An}$ interesting finding is that both increased and decreased MyD88 activity in Paneth cells modulates the number of translocating bacteria, but does not change the luminal bacterial density. Bacterial density in the lumen is most likely restricted by other factors such as nutrient availability, peristalsis, epithelial cell turnover, and shedding. ${ }^{55}$ MyD88 ablation reduces Paneth cell RegIII $\gamma$ production, but has no effect on CRS4C-4 synthesis,
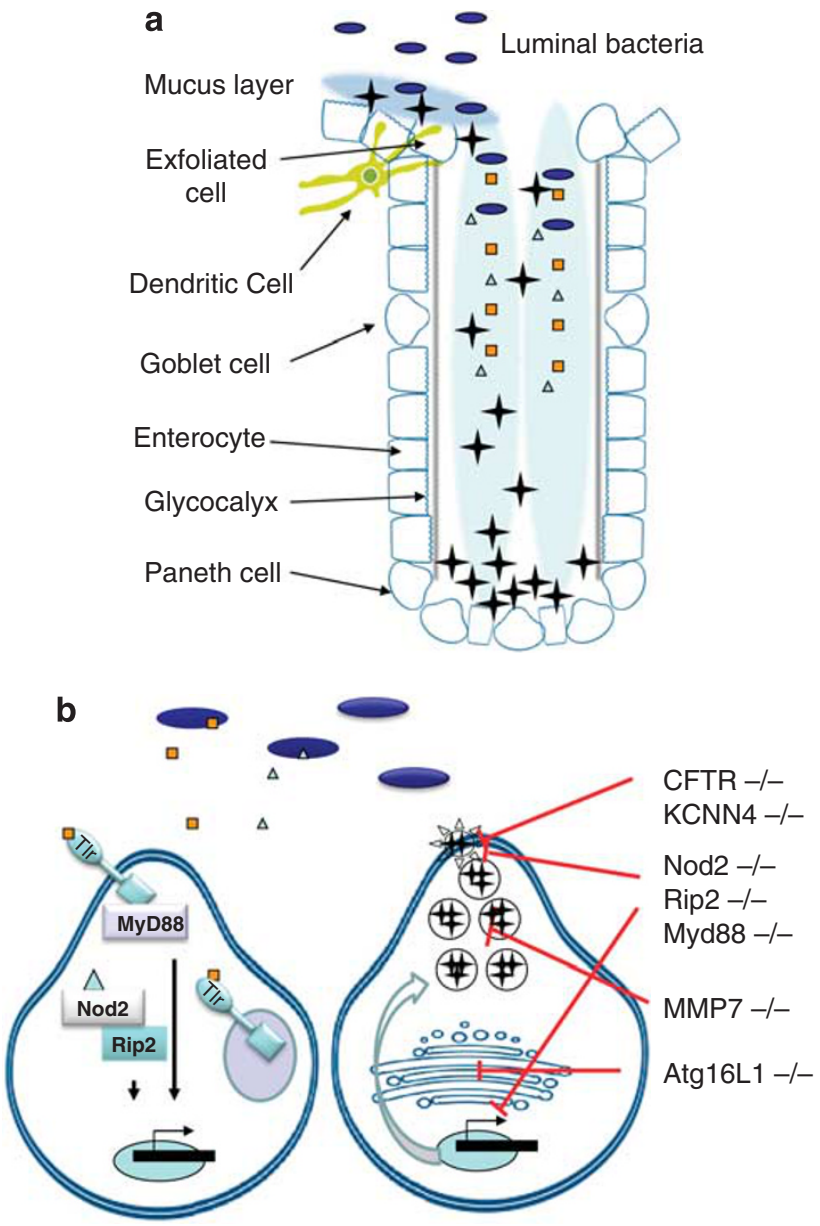

Figure 7 (a) Schematic representation of the small intestinal crypts of mice and man. Ovals represent bacteria, squares and triangles represent various microbial associated molecular pattern molecules (lipopolysaccharide (LPS), peptidoglycan fragments, lipopeptides, etc.), stars represent antimicrobial peptides/proteins. (b) Paneth cell microbial recognition and antimicrobial function. Left: Functional Paneth cells. Triangles and squares represent various microbial-associated molecular pattern molecules (LPS, peptidoglycan fragments, lipopeptides, etc.). Right: Lost Paneth cell functions as a consequence of various gene ablations/dysfunctions: pattern recognition receptor signaling (nucleotide-binding oligomerization domain-containing protein 2 (Nod2), myeloid differentiation primary response gene (88) (MyD88), receptor interacting protein-2 (Rip2)), granule secretion (CFTR, KCNN4), antimicrobial peptide processing (matrix metalloproteinase 7 (MMP7)), and Atg16L1 leading to Paneth cell autophagy. Lost functions are schematically shown as red T-lines. For reference to the different (-/-) mice, see text. TIr, Toll-like receptor.

underscoring that these Paneth cell products are differentially regulated.

(ii) MMP7-deficient mice (on a $\mathrm{C} 57 \mathrm{Bl} / 6$ genetic background) are unable to process $\alpha$-defensins and CRS-peptides to active peptides. These mice display no spontaneous intestinal inflammation, but instead they present with enhanced susceptibility to oral infection with E. coli or attenuated S. typhimurium. ${ }^{61,62}$ Transgenic co-expression of the human defensin HD5, which does not require MMP7 for activation, did complement the normal mouse repertoire of Paneth cell-derived $\alpha$-defensins/ 
CRS-peptides and enhanced the capacity to tolerate oral infection. ${ }^{63}$ This increased protection against Salmonella might not only have resulted from the introduced anti-Salmonella activity, but also be a consequence of an altered resident microflora (discussed below).

(iii) Paneth cell secretion involves a multisubunit $\mathrm{K}^{+}$pump that modulates $\mathrm{Ca}^{2+}$ fluxes. KCNN4 is one component of this multisubunit pump and the use of selective KCNN4 blockers markedly inhibited mouse Paneth cell secretion, as measured by the reduction in bactericidal activity and secreted antimicrobial peptides. ${ }^{64}$ Interestingly, this gene confers genetic susceptibility to the development of Crohn's ileal inflammation in humans. ${ }^{65}$

Malfunction of Atg16L1 induces autophagy in Paneth cells from man and mice. ${ }^{65}$ In mice, this abnormality of Paneth cells is induced by enteric norovirus and further modulated by tumor-necrosis- $\alpha$, interferon- $\gamma$, as well as commensal bacteria. ${ }^{66}$ In humans, mutations in the ATG16L1 gene have been linked to Crohn's disease. In another model, Paneth cell ablation can be induced in mice by the expression of a toxin under regulation by a $\alpha$-defensin promoter. These mice display no difference in their luminal bacteria density, but show increased levels of commensal bacteria in their mesenteric lymph nodes. This bacterial translocation indicates that Paneth cell-ablated mice are unable to protect the epithelial layer from invasion. ${ }^{55}$

In the cystic fibrosis mouse model, reduced $\alpha$-defensin and CRS activity facilitates infection with Salmonella via oral inoculation. These mice have a skewed Paneth cell function as a result of the defective cystic fibrosis transmembrane conductance regulator. Their Paneth cells have reduced antimicrobial secretions owing to limited dissolution of the granules in the lumen, and consequently there is an accumulation of granules containing trapped $\alpha$-defensins and CRS-peptides within the crypts. $^{67}$

The genetically modified mice presented above, all of which affect Paneth cell function, mirror certain gene defects in human inflammatory bowels diseases. Other mouse strains develop spontaneously intestinal inflammation that in turn distorts Paneth cell function. The Samp/YitFc mouse is known to develop spontaneous ileitis ${ }^{61}$ and has normal expression of $\alpha$-defensins and CRS4C-4 prior to inflammation. Interestingly, the transcript of CRS4C-4 is dramatically reduced during the chronic phase of inflammation in contrast to $\alpha$-defensin mRNA, which remained unaffected in the chronic phase. This profile of CRS4C- 4 bears resemblance to what is observed in Crohn's ileitis, in which the level of HD5 and HD6 is clearly reduced in affected intestines. ${ }^{57}$

A plausible model that connects Paneth cell secretions and intestinal inflammation suggests that Paneth cell antimicrobial factors regulate the number of bacteria that are in close proximity to the mucosal surface ${ }^{68}$ (Figure 7a). This idea is consistent with the fact that secreted Paneth cell antibacterial factors are retained by the mucus layer that lines the intestinal epithelium, but are virtually absent from luminal content. ${ }^{9}$ The mucus barrier may thus define a confined space that allows the host to specifically monitor and regulate bacteria that are in close contact with the intestinal surface. ${ }^{69}$ This model is further supported by the finding that Paneth cells sense bacteria that closely associate with the mucosal surface, which results in activation of MyD88-dependent antimicrobial responses, but are insensitive to the overall luminal bacterial load. ${ }^{55}$

\section{Effect of the normal flora}

Antimicrobial substances secreted from Paneth cells and epithelial cells are believed to "sterilize" the lower parts of the crypt in the healthy gut. Recent data demonstrate, in addition, a role for these peptides in shaping the endogenous microflora. ${ }^{70}$ The enteric antimicrobial peptides probably also inhibit bacteria from attaching to the intestinal epithelial monolayer. Thus, the influence of antimicrobial peptides on the local microflora and consequently the intrinsic self-regulatory function of the microflora should not be underestimated. Mice (FvB background) with enhanced $\alpha$-defensin production through the insertion of an enterically-directed human HD5 gene display a shift in the composition of the intestinal microflora, from predominantly bacteria of the Firmicutes phylum in the wild-type mice to a population that is dominated by the Bacteroidetes phylum in the HD5-expressing mice. ${ }^{70}$ On the contrary, the MMP7-deficient mice ( $\mathrm{C} 57 \mathrm{Bl} / 6$ background), with a reduced $\alpha$-defensin level, present a reduced number of bacteria of the Bacteroidetes phylum as compared with the parental strain. The two mouse strains display thus bacterial phyla differences, but the total number of bacteria that form the microflora is not changed in the HD5- or MMP7-modified mice. ${ }^{70}$ The most important change in HD5 transgenic mice is the lack of segmented filamentous bacteria, a bacterium known to reach direct contact with the intestinal epithelium. These data from the genetically modified mice parallel the findings in patients with Crohn's disease, who demonstrate reduced levels of $\alpha$-defensins, ${ }^{57}$ bacteria in close contact to the epithelial layers of inflamed patches in the small intestine, ${ }^{71}$ and an altered microflora, including overrepresentation of a specific bacterial species, Faecalibacterium prausnitzii. $^{72}$

\section{CONCLUSIONS}

The CRS-peptides family has so far been found exclusively in mice and extensive comparative analysis has not revealed any conclusive matches to the $3^{\prime} \mathrm{C}$-terminal end of the genes encoding the antimicrobially active part of CRS-peptides. ${ }^{19,73}$ It is an intriguing question why mice have invested in this unique and extensive family of peptides with such a specific amino-acid composition and high content of cysteines. The high expression level in terms of produced peptides argues in favor of a strong selection pressure to maintain this specific peptide family. Mouse neutrophils lack $\alpha$-defensins, whereas rat, rabbit, and man all produce myeloid $\alpha$-defensins. It may be that mice have compensated for this deficiency in neutrophil armament by an expansion of the Paneth cell antimicrobial repertoire through production of a wealth of CRS-peptides - which, by dimerization, expand the antimicrobial repertoire even further. The unusual covalent dimerization of CRS-peptides calls 
for further investigation into their biochemical characteristics and biological function, relating to the following questions: (i) Is more than one pair of cysteine bonds involved in the dimerization? (ii) At what point during CRS-peptide production does the dimerization occur? (iii) Are there any usher type of proteins required to assist specific dimerization or is it a random process? (iv) What are the structural entities in the various peptide dimers that give rise to differences in antimicrobialactivity? (v) Does the high content of cysteine bridges that potentially could be reduced have an impact on the redox status in the intestine?

The high ileal expression of CRS4C peptides in adult animals suggests not only a significant role in the control of the ileal microbiota, but also a role in protecting against ascending cecal bacteria. This differential longitudinal expression pattern of Paneth cell antimicrobial peptides is similar in mice and man, as is the regulation of $\alpha$-defensin transcription by the transcription factor Tcf- $4 .{ }^{42}$ The possibility of using mouse models to explore the cause of ileitis has improved during the past years thanks to the emerging insight into innate response receptors to bacterial components via gene knockouts and into the details of the intestinal microflora composition. ${ }^{74}$ The spectrum of differentially expressed CRS-peptides and $\alpha$-defensins in commonly used mouse strains offers additional avenues of investigations when used as tools to unravel the details of peptide-mediated defense and its role in gut homeostasis.

\section{ACKNOWLEDGMENTS}

The authors are grateful to Charles B. Bevins for fruitful discussions. This study was supported by grants from the Swedish Research Council, Ruth and Richard Juhlins Stiftelse, Socialstyrelsens fonder, and the Swedish Foundation for International Cooperation in Research and Higher Education (STINT).

\section{DISCLOSURE}

The authors declared no conflict of interest.

\section{() 2012 Society for Mucosal Immunology}

\section{REFERENCES}

1. Velagapudi, V.R. et al. The gut microbiota modulates host energy and lipid metabolism in mice. J. Lipid Res. 51, 1101-1112 (2010).

2. Backhed, F. et al. The gut microbiota as an environmental factor that regulates fat storage. Proc. Natl. Acad. Sci. USA 101, 15718-15723 (2004).

3. Cosnes, J., Gower-Rousseau, C., Seksik, P. \& Cortot, A. Epidemiology and natural history of inflammatory bowel diseases. Gastroenterology 140, 1785-1794 (2011).

4. Porter, E.M., Bevins, C.L., Ghosh, D. \& Ganz, T. The multifaceted Paneth cell. Cell Mol. Life Sci. 59, 156-170 (2002).

5. Crosnier, C., Stamataki, D. \& Lewis, J. Organizing cell renewal in the intestine: stem cells, signals and combinatorial control. Nat. Rev. Genet. 7, 349-359 (2006).

6. Ayabe, T. et al. Secretion of microbicidal alpha-defensins by intestinal Paneth cells in response to bacteria. Nat. Immunol. 1, 113-118 (2000).

7. Satoh, Y. Atropine inhibits the degranulation of Paneth cells in ex-germfree mice. Cell Tissue Res. 253, 397-402 (1988).

8. Ayabe, T. et al. Activation of Paneth cell alpha-defensins in mouse small intestine. J. Biol. Chem. 277, 5219-5228 (2002)

9. Meyer-Hoffert, U. et al. Secreted enteric antimicrobial activity localises to the mucus surface layer. Gut 57, 764-771 (2008).
10. Mastroianni, J.R. \& Ouellette, A.J. Alpha-defensins in enteric innate immunity: functional Paneth cell alpha-defensins in mouse colonic lumen. J. Biol. Chem. 284, 27848-27856 (2009).

11. Hill, C.P., Yee, J., Selsted, M.E. \& Eisenberg, D. Crystal structure of defensin HNP-3, an amphiphilic dimer: mechanisms of membrane permeabilization. Science 251, 1481-1485 (1991).

12. Zimmermann, G.R., Legault, P., Selsted, M.E. \& Pardi, A. Solution structure of bovine neutrophil beta-defensin-12: the peptide fold of the beta-defensins is identical to that of the classical defensins. Biochemistry 34, 13663-13671 (1995).

13. Huttner, K.M., Selsted, M.E. \& Ouellette, A.J. Structure and diversity of the murine cryptdin gene family. Genomics 19, 448-453 (1994).

14. Selsted, M.E., Miller, S.I., Henschen, A.H. \& Ouellette, A.J. Enteric defensins: antibiotic peptide components of intestinal host defense. J. Cell Biol. 118, 929-936 (1992).

15. Ouellette, A.J. et al. Mouse paneth cell defensins: primary structures and antibacterial activities of numerous cryptdin isoforms. Infect. Immun. 62, 5040-5047 (1994).

16. Shanahan, M.T., Tanabe, H. \& Ouellette, A.J. Paneth cell alpha-defensins in C57BL/6 mice: charaterization of strain-specific peptide and gene polymorphisms and vestigal myeloid alpha-defensin pseudogenes. Infect. Immun. 79, 459-473 (2010).

17. Ouellette, A.J. \& Lualdi, J.C. A novel mouse gene family coding for cationic, cysteine-rich peptides - regulation in small-intestine and cells of myeloid origin. J. Biol. Chem. 265, 9831-9837 (1990).

18. Hornef, M.W., Pütsep, K., Karlsson, J., Refai, E. \& Andersson, M. Increased diversity of intestinal antimicrobial peptides by covalent dimer formation. Nat. Immunol. 5, 836-843 (2004).

19. Amid, C. et al. Manual annotation and analysis of the defensin gene cluster in the C57BL/6J mouse reference genome. BMC Genomics 10, 606 (2009).

20. Karlsson, J. et al. Regional variations in Paneth cell antimicrobial peptide expression along the mouse intestinal tract. BMC Immunol. 9, 37 (2008).

21. Huttner, K.M. \& Ouellette, A.J. A family of defensin-like genes codes for diverse cysteine-rich peptides in mouse Paneth cells. Genomics 24, 99-109 (1994).

22. Ouellette, A.J., Pravtcheva, D., Ruddle, F.H. \& James, M. Localization of the cryptdin locus on mouse chromosome 8. Genomics 5, 233-239 (1989).

23. Groth, M. et al. High-resolution mapping of the 8p23.1 beta-defensin cluster reveals strictly concordant copy number variation of all genes. Hum. Mutat. 29, 1247-1254 (2008).

24. Henrichsen, C.N. et al. Segmental copy number variation shapes tissue transcriptomes. Nat. Genet. 41, 424-429 (2009).

25. Sudmant, P.H. et al. Diversity of human copy number variation and multicopy genes. Science 330, 641-646 (2010).

26. Morrison, G.M., Semple, C.A., Kilanowski, F.M., Hill, R.E. \& Dorin, J.R. Signal sequence conservation and mature peptide divergence within subgroups of the murine beta-defensin gene family. Mol. Biol. Evol. 20, 460-470 (2003).

27. Semple, C.A. et al. The complexity of selection at the major primate betadefensin locus. BMC Evol. Biol. 5, 32-46 (2005).

28. Hollox, E.J. \& Armour, J.A. Directional and balancing selection in human beta-defensins. BMC Evol. Biol. 8, 113-127 (2008).

29. Weeks, C.S. et al. Matrix metalloproteinase-7 activation of mouse paneth cell pro-alpha-defensins: SER43/ILE44 proteolysis enables membrane-disruptive activity. J. Biol. Chem. 281, 28932-28942 (2006).

30. Ghosh, D. et al. Paneth cell trypsin is the processing enzyme for human defensin-5. Nat. Immunol. 3, 583-590 (2002).

31. Pütsep, K. et al. Germ-free and colonized mice generate the same products from enteric prodefensins. J. Biol. Chem. 275, 40478-40482 (2000).

32. Shirafuji, Y. et al. Structural determinants of procryptdin recognition and cleavage by matrix metalloproteinase-7. J. Biol. Chem. 278, 7910-7919 (2003).

33. Grandjean, V., Vincent, S., Martin, L., Rassoulzadegan, M. \& Cuzin, F. Antimicrobial protection of the mouse testis: synthesis of defensins of the cryptdin family. Biol. Reprod. 57, 1115-1122 (1997).

34. Com, E. et al. Expression of antimicrobial defensins in the male reproductive tract of rats, mice, and humans. Biol. Reprod. 68, 95-104 (2003). 
35. Jelinsky, S.A. et al. The rat epididymal transcriptome: comparison of segmental gene expression in the rat and mouse epididymides. Biol. Reprod. 76, 561-570 (2007).

36. Wehkamp, J. et al. Paneth cell antimicrobial peptides: topographical distribution and quantification in human gastrointestinal tissues. FEBS Lett. 580, 5344-5350 (2006).

37. Sundbom, M. et al. Alteration in human defensin- 5 expression following gastric bypass surgery. J. Clin. Pathol. 60, 1029-1034 (2007).

38. Hauck, A.L. et al. Twists and turns in the development and maintenance of the mammalian small intestine epithelium. Birth Defects Res. C Embryo Today 75, 58-71 (2005).

39. van Es, J.H. et al. Wnt signalling induces maturation of Paneth cells in intestinal crypts. Nat. Cell Biol. 7, 381-386 (2005).

40. Korinek, V. et al. Constitutive transcriptional activation by a beta-cateninTcf complex in APC-/- colon carcinoma. Science 275, 1784-1787 (1997).

41. Andreu, P. et al. Crypt-restricted proliferation and commitment to the Paneth cell lineage following Apc loss in the mouse intestine. Development 132, 1443-1451 (2005).

42. Wehkamp, J. et al. The Paneth cell alpha-defensin deficiency of ileal Crohn's disease is linked to Wnt/Tcf-4. J. Immunol. 179, 3109-3118 (2007).

43. Bry, L. et al. Paneth cell differentiation in the developing intestine of normal and transgenic mice. Proc. Natl. Acad. Sci. USA 91, 10335-10339 (1994).

44. Darmoul, D., Brown, D., Selsted, M.E. \& Ouellette, A.J. Cryptdin gene expression in developing mouse small intestine. Am. J. Physiol. 272, G197-206 (1997).

45. Menard, S. et al. Developmental switch of intestinal antimicrobial peptide expression. J. Exp. Med. 205, 183-193 (2008).

46. Satoh, Y. Effect of live and heat-killed bacteria on the secretory activity of Paneth cells in germ-free mice. Cell Tissue Res. 251, 87-93 (1988).

47. Cash, H.L., Whitham, C.V., Behrendt, C.L. \& Hooper, L.V. Symbiotic bacteria direct expression of an intestinal bactericidal lectin. Science 313, 1126-1130 (2006).

48. Campopiano, D.J. et al. Structure-activity relationships in defensin dimers: a novel link between beta-defensin tertiary structure and antimicrobial activity. J. Biol. Chem. 279, 48671-48679 (2004).

49. Ouellette, A.J., Satchell, D.P., Hsieh, M.M., Hagen, S.J. \& Selsted, M.E. Characterization of luminal paneth cell alpha-defensins in mouse small intestine. Attenuated antimicrobial activities of peptides with truncated amino termini. J. Biol. Chem. 275, 33969-33973 (2000).

50. Schroeder, B.O. et al. Reduction of disulphide bonds unmasks potent antimicrobial activity of human beta-defensin 1 . Nature 469, 419-423 (2011).

51. Bjorkhem-Bergman, L., Jonsson-Videsater, K., Paul, C., Bjornstedt, M. \& Andersson, M. Mammalian thioredoxin reductase alters cytolytic activity of an antibacterial peptide. Peptides 25, 1849-1855 (2004).

52. Andersson, M., Holmgren, A. \& Spyrou, G. NK-lysin, a disulfidecontaining effector peptide of T-lymphocytes, is reduced and inactivated by human thioredoxin reductase. Implication for a protective mechanism against NK-lysin cytotoxicity. J. Biol. Chem. 271, 10116-10120 (1996).

53. Andersson, M. et al. NK-lysin, a novel effector peptide of cytotoxic T and NK cells. Structure and cDNA cloning of the porcine form, induction by interleukin 2, antibacterial and antitumour activity. EMBO J. 14, 1615-1625 (1995).

54. Winter, S.E. et al. Gut inflammation provides a respiratory electron acceptor for Salmonella. Nature 467, 426-429 (2010).

55. Vaishnava, S., Behrendt, C.L., Ismail, A.S., Eckmann, L. \& Hooper, L.V. Paneth cells directly sense gut commensals and maintain homeostasis at the intestinal host-microbial interface. Proc. Natl. Acad. Sci. USA 105, 20858-20863 (2008).
56. Biswas, A. et al. Induction and rescue of Nod2-dependent Th1-driven granulomatous inflammation of the ileum. Proc. Natl. Acad. Sci. USA 107, 14739-14744 (2010)

57. Wehkamp, J. et al. Reduced Paneth cell alpha-defensins in ileal Crohn's disease. Proc. Natl. Acad. Sci. USA. 102, 18129-18134 (2005).

58. Rehman, A. et al. Nod2 is essential for temporal development of intestinal microbial communities. Gut 60, 1354-1362 (2011).

59. Tanabe, H. et al. Mouse paneth cell secretory responses to cell surface glycolipids of virulent and attenuated pathogenic bacteria. Infect. Immun. 73, 2312-2320 (2005).

60. Lotz, M. et al. Postnatal acquisition of endotoxin tolerance in intestinal epithelial cells. J. Exp. Med. 203, 973-984 (2006).

61. Shanahan, M.T. et al. Elevated expression of Paneth cell CRS4C in ileitisprone SAMP1/YitFc mice: regional distribution, subcellular localization, and mechanism of action. J. Biol. Chem. 285, 7493-7504 (2010).

62. Wilson, C.L. et al. Regulation of intestinal alpha-defensin activation by the metalloproteinase matrilysin in innate host defense. Science $\mathbf{2 8 6}$, 113-117 (1999).

63. Salzman, N.H., Ghosh, D., Huttner, K.M., Paterson, Y. \& Bevins, C.L. Protection against enteric salmonellosis in transgenic mice expressing a human intestinal defensin. Nature 422, 522-526 (2003).

64. Ayabe, T. et al. Modulation of mouse Paneth cell alpha-defensin secretion by mIKCa1, a Ca2+-activated, intermediate conductance potassium channel. J. Biol. Chem. 277, 3793-3800 (2002).

65. Simms, L.A. et al. KCNN4 gene variant is associated with ileal Crohn's Disease in the Australian and New Zealand population. Am. J. Gastroenterol. 105, 2209-2217 (2010).

66. Cadwell, K. et al. A key role for autophagy and the autophagy gene Atg16l1 in mouse and human intestinal Paneth cells. Nature 456, 259-263 (2008).

67. Clarke, L.L. et al. Abnormal Paneth cell granule dissolution and compromised resistance to bacterial colonization in the intestine of CF mice. Am. J. Physiol. Gastrointest. Liver Physiol. 286, G1050-1058 (2004).

68. Salzman, N.H., Underwood, M.A. \& Bevins, C.L. Paneth cells, defensins, and the commensal microbiota: a hypothesis on intimate interplay at the intestinal mucosa. Semin. Immunol. 19, 70-83 (2007).

69. van der Waaij, L.A. et al. Bacterial population analysis of human colon and terminal ileum biopsies with $16 \mathrm{~S}$ rRNA-based fluorescent probes: commensal bacteria live in suspension and have no direct contact with epithelial cells. Inflamm. Bowel Dis. 11, 865-871 (2005).

70. Salzman, N.H. et al. Enteric defensins are essential regulators of intestinal microbial ecology. Nat. Immunol. 11, 76-83 (2010).

71. Darfeuille-Michaud, A. et al. High prevalence of adherent-invasive Escherichia coli associated with ileal mucosa in Crohn's disease. Gastroenterology 127, 412-421 (2004).

72. Willing, B. et al. Twin studies reveal specific imbalances in the mucosaassociated microbiota of patients with ileal Crohn's disease. Inflamm. Bowel Dis. 15, 653-660 (2009).

73. Patil, A., Hughes, A.L. \& Zhang, G. Rapid evolution and diversification of mammalian alpha-defensins as revealed by comparative analysis of rodent and primate genes. Physiol. Genomics 20, 1-11 (2004).

74. Friswell, M.K. et al. Site and strain-specific variation in gut microbiota profiles and metabolism in experimental mice. PLOS ONE 5, e8584 (2010).

This work is licensed under the Creative Commons Attribution-NonCommercial-No Derivative Works 3.0 Unported License. To view a copy of this license, visit http://creativecommons.org/licenses/by-nc-nd/3.0/ 